\title{
Assessment of Hand-surface Touching Behavior among Individuals Visiting Dental Hospital in Gurugram, Haryana: A Pilot Observational Study
}

\author{
Amit Bhardwaj ${ }^{1}$, Anurag Bhatnagar ${ }^{2}$, Shalu Verma ${ }^{3}$, Charu Khurana $^{4}$
}

\begin{abstract}
Aim and objective: This study aims to find out the frequency for touching the various surfaces including mucosal and non-mucosal as well as the use of preventive measures from coronavirus disease-2019 (COVID-19) among individuals visiting the dental hospital in Gurugram, Haryana. Materials and methods: A total of 34 individuals were observed over a period of 21 days for 60 minutes and the frequencies of their hand touching the various surfaces were recorded.

Results: The result showed a higher frequency of touching the surfaces in females. Mucosal and non-living surfaces were more touched than non-mucosal surfaces.

Conclusion: This study indicates that citizens must grab better concepts of infection spread to minimize the pandemic effect.

Clinical significance: Checking self-inoculation behavior can be a method of prevention of disease spread in dental hospitals.

Keywords: Behavior, COVID-19, Dental hospital, Hand and face touching, Inoculation, Surface touching, Touching

World Journal of Dentistry (2021): 10.5005/jp-journals-10015-1809
\end{abstract}

\section{INTRODUCTION}

A substantial portion of human respiratory tract infections (RTIs) is transmitted via contaminated hand contact by means of self-inoculation. ${ }^{1,2}$ Self-inoculation from the hand to the mucus membranes of the eyes, nose, and mouth is the common and most frequent method of transmission of RTIs. ${ }^{3}$ Although the literature on the mechanisms of self-inoculation of common respiratory infections (e.g., influenza, coronavirus) is limited, contaminated hands have been reported as having the potential to disseminate respiratory infections. ${ }^{2}$ Also, many synthetic viruses, rhinovirus, and anecdotal reports are transmitted by touching the mucosal surfaces of the face with contaminated hands. ${ }^{1}$ Therefore, recommendations to decrease the spread of RTIs include hand hygiene, i.e., either washing hands with soap and water or using an alcohol-based cleanser. ${ }^{3}$ A meta-analysis of eight selected intervention studies geared toward the general public showed a $24 \%$ decrease in respiratory illness relative to control groups due to hand-washing measures. ${ }^{1}$

A critical element in reducing transmission of any infectious respiratory disease is the behavior changes among the common people. ${ }^{4}$ Government and health agencies like the World Health Organization, Ministry of Health and Family Welfare, India have been trying to implement the behavior changes in the citizens since the early days of the coronavirus disease-2019 (COVID-19) crisis. ${ }^{5,6}$ Personal protective measures like hand washing, respiratory mask, social distancing, and minimum/no touching of the face are effective in few observational studies worldwide. ${ }^{4}$

During the time of the COVID-19 pandemic, the hand-face touching behavior in the potential infectious community has been observed. The frequency of the exposure to the potential carrier in community and healthcare settings is at high risk. In the healthcare setting, frequent face touching, particularly during periods of the seasonal outbreak, has the theoretical potential to be a mechanism of transmission and acquisition of the disease. ${ }^{1}$
1,2Department of Periodontology, Faculty of Dental Sciences, SGT
University, Gurugram, Haryana, India
${ }^{3}$ Department of Pedodontics and Preventive Dentistry, Faculty of
Dental Sciences, SGT University, Gurugram, Haryana, India
${ }^{4}$ Department of Public Health Dentistry, Faculty of Dental Sciences,
SGT University, Gurugram, Haryana, India Corresponding Author: Amit Bhardwaj, Department of Periodontology, Faculty of Dental Sciences, SGT University, Gurugram, Haryana, India, Phone: +91 9818718872, e-mail: amit.bhardwaj@ sgtuniversity.org

How to cite this article: Bhardwaj A, Bhatnagar A, Verma S, et al. Assessment of Hand-surface Touching Behavior among Individuals Visiting Dental Hospital in Gurugram, Haryana: A Pilot Observational Study. World J Dent 2021;12(2):121-125.

Source of support: Nil

Conflict of interest: None

Quantifying the hand and face touching in the spreading of RTIs is an hour of need to know the behavioral changes in the community after continuous implementation of personal protective measures by the health agencies.

To better understand these behavioral dynamics between surface, hand, and face touching among the community an initial initiative had been taken upon in the form of this study to find the prevalence of hand-to-surface touching among patients visiting the dental hospitals in Gurugram, Haryana (India).

\section{Materials and Methods}

This observational study was conducted in Level-2 Clinic (under the advisory of $\mathrm{DCl}$ instructions) ${ }^{7}$ between May 25 and June 15, 2020 (total days for study-21). The study was approved by the Ethical 
Committee of Institutional review board (SGTU/FDS/Perio/08/2020) before its commencement. Informed consent was obtained from the patients and those who agreed to this study were included.

\section{Participants}

This study was conducted with 34 patients ( 14 male and 20 female) aged 5-70 years from the dental outpatient department of SGT Dental College, Gurugram, Haryana, India. For further study purposes, the patients were also grouped based upon age: (1) Group I: 5-14 years; (2) Group II: 15-60 years; (3) Group III: >60 years.

Patients were observed for their hand and face touching behavior for 60 minutes while sitting in a well-ventilated waiting area. The patients were only informed about the observations being made for personal protective protocol. To limit the likelihood of changed behavior for hand-face touching, the patients were blindsided on this aspect to eliminate the bias.

\section{Data Collection}

To observe the hand and face touching, surfaces were divided into two categories: (1) Living surfaces which includes face comprising of mucosal surface (eyes, nose, and mouth) and non-mucosal surface (forehead, chin, cheeks, ears, and hair) and (2) Non-living surface which included metal (chair and door handle) and non-metal (glass, plastic/paper, wood and face mask). During the time of the study, a trained observer observed the surfaces that were touched by hand and the number of times the patients touched these surfaces. Also, the number of patients who entered the clinical area wearing a mask and practiced hand sanitization was observed.

\section{Data Analysis}

The observed data were deposited on a Microsoft Office Excel 2010 spreadsheet. Descriptive statistics were performed to determine the frequency distribution and duration of touching various surfaces per hour using SPSS version 21 software (SPSS Inc., Chicago, IL, USA). For better statistical analysis, the surface touching was further categorized into three categories based on the number of times it was touched per 60 minutes. It includes category I: 0-5 times, category II: 6-10 times, and category III: $>10$ times. Chi-square test was used for intergroup comparison at $p<0.05$ being significant.

\section{Results}

In this study, a total of 34 patients were observed out of which 14 were male with a mean age of $28.1 \pm 19.9$ years and 20 were female with a mean age of $29.9 \pm 20.7$ years. Out of 34 patients, 10 (29.41\%) belonged to group I (mean age $9.8 \pm 2.7$ years), 18 (52.94\%) belonged to group II (mean age $27.8 \pm 9.6$ years), and 6 (17.64\%) belonged to group III (mean age $65.3 \pm 3.1$ years) (Table 1).

The distribution of using face masks was seen among $82.3 \%$ of patients, with males (85.7\%) frequency higher than females (80\%) on entering the hospital. The distribution among the age group showed $100 \%$ use of masks in group III compared to group I (80\%) and group II (77.7\%). When compared within the groups, the values showed non-significant results ( $p>0.05)$ (Table 2$)$.

It was observed that the practice of hand sanitization was seen among $23.5 \%$ of patients, of which males (28.5\%) practiced more than females (20\%). About $76.4 \%$ of the patients did not abide by the use of hand sanitization upon entering the hospital. The distribution of hand sanitization practice in age group I was $20 \%$ and group II was $33.3 \%$. In group III, no one practiced hand-
Table 1: Demographic details of patients visiting the dental hospital $(n=34)$

\begin{tabular}{llc}
\hline Variable & $n(\%)$ & Mean age $\pm S D$ (years) \\
\hline Gender & & \\
$\quad$ Male & $14(41.1)$ & $28.1 \pm 19.9$ \\
$\quad$ Female & $20(58.8)$ & $29.9 \pm 20.7$ \\
Age (years) & & \\
5-14 (group I) & $10(29.4)$ & $9.8 \pm 2.7$ \\
15-60 (group II) & $18(52.9)$ & $27.8 \pm 9.6$ \\
$>60$ (group III) & $6(17.6)$ & $65.3 \pm 3.1$ \\
\hline
\end{tabular}

$n$ is the number of patients, \% is the percentage of patients, SD, standard deviation

Table 2: Distribution of patients using a face mask and hand sanitization on entering the dental hospital

\begin{tabular}{|c|c|c|c|c|}
\hline \multirow{2}{*}{$\begin{array}{l}\text { Category } \\
\text { Patients', } n \\
(\%) \\
\end{array}$} & \multicolumn{2}{|c|}{ Face mask } & \multicolumn{2}{|c|}{ Hand sanitization } \\
\hline & Yes & No & Yes & No \\
\hline \multicolumn{5}{|l|}{ Gender } \\
\hline Male & $12(85.7)$ & $02(14.2)$ & $4(28.57)$ & $10(71.4)$ \\
\hline Female & $16(80)$ & $04(20)$ & $4(20)$ & $16(80)$ \\
\hline Total & $28(82.3)$ & $06(17.6)$ & $8(23.5)$ & $26(76.4)$ \\
\hline$p$ & 0.66 (NS) & & 0.56 (NS) & \\
\hline \multicolumn{5}{|c|}{ Age groups $(n=34)$} \\
\hline Group I & $08(80)$ & $02(20)$ & $02(20)$ & $08(80)$ \\
\hline Group II & $14(77.7)$ & $04(22.2)$ & $06(33.3)$ & $12(66.6)$ \\
\hline Group III & $06(100)$ & 0 & 0 & $06(100)$ \\
\hline Total & $28(82.3)$ & $06(17.6)$ & $08(23.5)$ & $26(76.4)$ \\
\hline$p$ & 0.45 (NS) & & 0.18 (NS) & \\
\hline
\end{tabular}

Chi-square test; ${ }^{*} p \leq 0.05$ (significant), $n$ is the number of patients, $\%$ is the percentage of patients, NS; non-significant

sanitization. The results were non-significant when compared within the groups (Table 2).

A total of 588 hand touches were made over a period of 60 minutes including all living and non-living surfaces.

The face was touched 199 times which accounts for all living surfaces. Among these, the mucosal and non-mucosal surfaces alone were touched by $23.5 \%$ of individuals and $41.1 \%$ of individuals touched both the surfaces with non-significant results $p>0.05$ (Table 3 shows the male and female distribution). The frequency distribution of patients touching living surfaces (face) among the age groups showed a higher percentage in the patients touching both the surfaces (47.05\%) and within it, group III (66.6\%) was highest, followed by group II (44.4\%) and group I (40\%). Touching among mucosal and non-mucosal surface showed was $22.2 \%$ in group II and $0 \%$ in group III. In group I, the mucosa was touched by $20 \%$ and non-mucosal was touched by $40 \%$ of patients in 60 minutes (Table 3). The results were non-significant $p>0.05$.

Non-living surfaces were touched 389 times in 60 minutes. The metal alone was touched by $5.8 \%$ of individuals, non-metal surfaces alone were not touched at all while both metal and non-metal surfaces were touched by $94.1 \%$ of patients which were significant $p<0.05$ (Table 3 shows the male and female distribution). The frequency distribution of patients touching non-living surfaces among the age groups showed a higher percentage in the patients 
Hand-surface Touching Behavior in Dental Hospital

Table 3: Frequency distribution of dental patients touching living and non-living surfaces (type) in 60 minutes visiting a dental hospital

\begin{tabular}{|c|c|c|c|c|c|c|c|c|}
\hline \multirow{2}{*}{$\begin{array}{l}\text { Surface types } \\
n(\%)\end{array}$} & \multicolumn{4}{|c|}{ Living } & \multicolumn{4}{|c|}{ Non-living } \\
\hline & Mucosal & Non-mucosal & Both & Not touched & Mucosal & Non-mucosal & Both & Not touched \\
\hline \multicolumn{9}{|l|}{ Gender } \\
\hline Male & $2(14.2)$ & $6(42.8)$ & $2(28.5)$ & $2(14.2)$ & $2(14.2)$ & 0 & $12(85.7)$ & $6(42.8)$ \\
\hline Female & $6(30)$ & $2(10)$ & $10(50)$ & $2(10)$ & 0 & 0 & $20(100)$ & 0 \\
\hline Total & $8(23.5)$ & $8(23.5)$ & $14(41.1)$ & $4(11.7)$ & $2(5.8)$ & 0 & $32(94.1)$ & $6(17.6)$ \\
\hline$p$ & 0.61 (NS) & & & & $0.048^{*}(S)$ & & & \\
\hline \multicolumn{9}{|c|}{ Age groups $(n=34)$} \\
\hline Group I & $2(20)$ & $4(40)$ & $4(40)$ & 0 & $1(10)$ & 0 & $9(90)$ & 0 \\
\hline Group II & $4(22.2)$ & $4(22.2)$ & $8(44.4)$ & 0 & $1(5.5)$ & 0 & $17(94.4)$ & 0 \\
\hline Group III & 0 & 0 & $4(66.6)$ & $2(33.3)$ & 0 & 0 & $6(100)$ & 0 \\
\hline Total & $6(17.6)$ & $8(23.5)$ & $16(47.05)$ & $4(11.7)$ & $2(5.8)$ & 0 & $32(94.1)$ & 0 \\
\hline$p$ & 0.66 (NS) & & & & $0.001^{*}(\mathrm{HS})$ & & & \\
\hline
\end{tabular}

Chi-square test; ${ }^{*} p \leq 0.05$ (significant), $n$ is the number of patients, $\%$ is the percentage of patients, NS, non-significant; $S$, significant; HS, highly significant

Table 4: Frequency distribution of dental patients touching living and non-living surfaces (Category) in 60 minutes visiting dental hospital based on age groups

\begin{tabular}{|c|c|c|c|c|c|c|}
\hline \multirow{2}{*}{$\begin{array}{l}\text { Surface } \\
\text { Frequency of } \\
\text { touching, } n(\%)\end{array}$} & \multicolumn{3}{|c|}{ Living } & \multicolumn{3}{|c|}{ Non-living } \\
\hline & Category I & Category II & Category III & Category I & Category II & Category III \\
\hline \multicolumn{7}{|l|}{ Gender } \\
\hline Male & $12(85.7)$ & $2(14.2)$ & 0 & $4(28.5)$ & $4(28.5)$ & $6(42.8)$ \\
\hline Female & $6(30)$ & $8(40)$ & $6(30)$ & $3(15)$ & $4(20)$ & $13(65)$ \\
\hline Total & $18(52.9)$ & $10(29.4)$ & $6(17.6)$ & $7(20.5)$ & $8(23.5)$ & $19(55.8)$ \\
\hline$p$ & 0.08 (NS) & & & $0.043^{*}(\mathrm{~S})$ & & \\
\hline \multicolumn{7}{|c|}{ Age groups $(n=34)$} \\
\hline Group I & $6(60)$ & $3(30)$ & $1(10)$ & $1(10)$ & $2(20)$ & $7(70)$ \\
\hline Group II & $11(61.11)$ & $3(16.66)$ & $4(22.22)$ & $5(27.78)$ & $6(33.33)$ & $7(38.89)$ \\
\hline Group III & $2(33.33)$ & $3(50)$ & $1(16.66)$ & $1(16.66)$ & 0 & $5(83.33)$ \\
\hline Total & $19(55.8)$ & $9(26.47)$ & $6(17.6)$ & $7(20.5)$ & $8(23.5)$ & $19(55.8)$ \\
\hline$p$ & 0.57 (NS) & & & $0.04 *(S)$ & & \\
\hline
\end{tabular}

Chi-square test; ${ }^{*} p \leq 0.05$ (significant), $n$ is the number of patients, \% is the percentage of patients, frequency of Touching-Category I: $0-5$ times, Category II: 6-10 times and Category III: >10 times, NS, non-significant; S, significant

touching both the surfaces (94.1\%) and within it, group III (100\%) was highest, followed by group II (94.4\%) and group I (90\%). There was no touching of surfaces among non-metal and group III metal groups. However, metal alone was touched by $10 \%$ in group I and $5.5 \%$ in group II (Table 3 ). The results were highly significant $p>$ 0.05 within the groups.

Among the living surfaces (face), the frequency of touching was higher in category I (52.9\%) wherein males (85.7\%) touched more surfaces than females (30\%) per 60 minutes, followed by category II (29.4\%) and category III (17.6\%). Age groups also followed a similar pattern with more in category I (55.8\%) wherein group II (61.1\%) was highest compared to group I (60\%) and group III (33.33\%), followed by category II (26.4\%) and category III (17.8\%). The intergroup comparison showed non-significant results $p>0.05$ (Table 4).

However, the frequency of touching non-living surfaces per 60 minutes showed a statistically significant result $(p<0.05)$. Category III (55.8\%) showed the highest number of touching metal-nonmetal surfaces, in which females (65\%) and age group III (83.8\%) touched more surfaces than males (42.8\%) and age group I (70\%) and group II (38.89\%). Followed by category II (23.5\%) in which males (28.5\%) and age group II (33.3\%) touched more surfaces than females (20\%) and age group I (20\%). In category I (20.5\%), in which males $(28.5 \%)$ and age group II (27.7\%) touched more surfaces than females (15\%) and age group III (16.6\%), and group I (10\%) touched the surfaces (Table 4).

Also, the rate of touching mucosal surfaces on an average per 60 minutes, for the eyes were 2.1 times, the nose was 5.2 times, and the mouth was 3.56 times. The non-mucosal surfaces were touches on an average, the forehead was 1.3 times, the cheeks were 1.6 times, the chin was 1.62 times, the ears were 1 time, and the hair was 2.5 times. Similarly, the average number of times metal surfaces were touched, the chair was 2.5 times, and the door handle was 2.8 times. The non-metal surfaces were touched, the glass was 2.09 times, the wood was 4.25 times, the plastic and papers was 2.7 times, and the mask that was worn by the patient was 4.3 times (Fig. 1).

\section{Discussion}

COVID-19 is a serious disease that spreads through infected respiratory droplets or by contact with such materials. ${ }^{8}$ Coughing and sneezing by an infected person can cause a spread of SARSCoV-2 into the air, so those who are infected are a potential source 


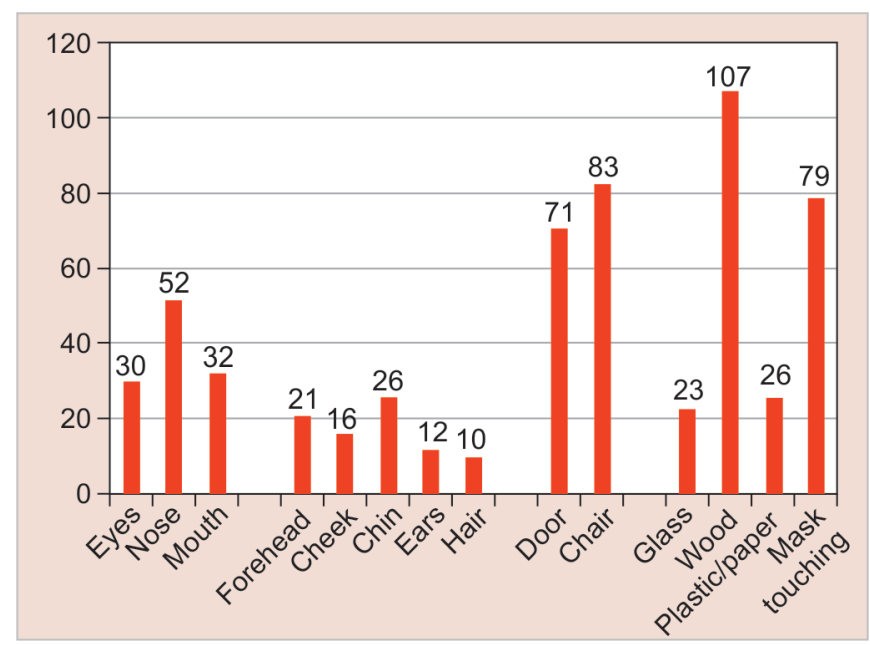

Fig. 1: Average number of times the surfaces were touched in 60 minutes

of spreading the infection to the healthy individuals coming in close contact (within a radius of approximately 6 feet). This led to the recent recommendation of social distancing to minimize the community spread of the disease. Droplets are an important route of transmission from inanimate objects located nearby to an infected person and which are subsequently touched by others. ${ }^{8}$ Therefore, hands are considered as a vector for the transmission of COVID, particularly during the prodromal stages of infection. The habitual behavior of hand-to-face touching has been observed in individuals with the possibility of self-inoculation. ${ }^{2}$ On average, an individual touches its face 3-5.3 times per hour. ${ }^{1,2}$ Keeping this into account, this study was designed to evaluate this type of behavior in individuals despite constant reminders from the government and other health services.

In this study, the frequency of touching the face was found to be within the range of 1-5.2 times per hour. This rate of touching was found to be higher in the Indian scenario as compared to the Japanese population. ${ }^{4}$ The Japanese model showed $40 \%$ of participants touching the face (eyes, nose, and mouth). This difference is although because countries like Japan have been practicing the habit of wearing a mask for many years due to the higher frequency of respiratory diseases ${ }^{4}$ in their country compared to the Indian scenario.

Also, behavior related to personal protective measures was noted in the individuals coming to the dental hospital. The male population showed a better chance in the behavior than females when hand sanitation and wearing of the mask were observed. Within different age groups, hand sanitization was better performed by 15-60 years followed by $5-14$ years group with higher practice in males than females. The use of mask was embraced by older age group (above 60 years) in males compared to females than the 15-60 years age group which was embraced more in males than females. These differences may be due to differences in the level of awareness among the individuals. There is limited literature that supports the use of wearing the mask and practicing hand sanitization in reducing the transmission of the disease, but there are data on the mechanical model basis for protective measures to work. ${ }^{9-13}$ Although the implementation of protective measures has been developed at the time of pandemic in the general citizens of the nation, still one has to go a long way to change the lifestyle to combat the scenario.
In the study, a higher frequency of hand touching was seen in non-living (face) surfaces followed by living surfaces. Touching of metal and non-metal surfaces was observed more in females and older age groups compared to males and younger individuals. A similar pattern was observed in touching mucosal-non-mucosal surfaces.

The nose and mouth were the most common mucosal surfaces that were touched by hand at an average frequency per hour of 5.2 and 3.5, respectively, which is consistent with the study by Nicas and Best. ${ }^{1}$ Among non-mucosal surfaces which was least touched, hair was touched on an average of 2.5 times per hour followed by the chin, cheek, and forehead that were touched at an average of 1 time per hour. The average frequency of touching the inanimate surfaces was similar to mucosal surfaces. Touching of wooden desk, door handles, and mask (approximately 4 times per hour) was higher than touching metallic chair (2.5 times per hour).

The categorical classification gives a better idea of the frequency of touching. Accordingly in this study, patients have touched their faces at the rate of $0-5$ times per 60 minutes. This was commonly seen in males and 15-60 year age groups. And non-living surfaces were touched at a rate of $>10$ times per 60 minutes, commonly seen in males and older age groups. This strongly signifies the abovementioned statement. However, there are no studies to substantiate the distribution among genders and age groups. This study is first as far as the literature search suggests otherwise.

This clearly shows the habit of touching the face is prevalent among the population group despite repeated warnings and measures proposed by the health bodies. The possibility of selfinoculation through these contaminated surfaces especially the face mask that is worn by the individual may result as a vector to transmit disease into the respiratory tract. ${ }^{14}$ From this study, a small picture is clear that the population must be made more aware of the factors of the implication of the face touching behavior. Hence, proper hand hygiene practice and wearing masks in public places are essential tools in preventing the spread of the virus and also are inexpensive methods to break the cycle of transmission. The Centre for Disease Control also has stated that the avoidance of touching the eyes, nose, and mouth is useful to prevent the flu. ${ }^{15}$ This fact is also supported by Kwok et al., Machida et al., and Elder et al., where they all supported the incorporation of personal protective measures can protect the individual from disease transmission. ${ }^{2-4}$

There are some limitations to this study. First, only a limited geographic area, ethnic and culture was under behavioral observation as it might differ from region to region being India a diverse country. Second, patients who visited the dental hospital during the time of the COVID crisis were those who were in great need of dental treatment and visited the dental hospital despite strict guidelines from the state and dental council of India. Therefore, the patients recruited in the study were limited. Against all these limitations, to our best knowledge, this study is the first to highlight data on the behavioral pattern of the current population during the time of the COVID-19 pandemic.

\section{Conclusion}

It is clear from the study that a better implementation method has to be adopted by the population to prevent COVID-19 spread. The citizens must abide by changes at the early stage of the epidemic state rather than in the later pandemic stage. 
Few suggestions can be laid for hospitals:

- Keep proper charts on display for patient education.

- Use proper audio-visual aids in waiting areas to spread awareness.

- Small skits can be performed to enlighten on precautionary measures following social distancing.

\section{References}

1. Nicas $M$, Best D. A study quantifying the hand-to-face contact rate and its potential application to predicting respiratory tract infection. J Occup Environ Hyg 2008;5(6):347-352. DOI: 10.1080/15459620802003896.

2. Kwok YL, Gralton J, McLaws ML. Face touching: a frequent habit that has implications for hand hygiene. Am J Infect Control 2015;43(2):112 114. DOI: 10.1016/j.ajic.2014.10.015.

3. Elder NC, Sawyer W, Pallerla H, et al. Hand hygiene and face touching in family medicine offices: a Cincinnati area research and improvement group (CARInG) network study. J Am Board Fam Med 2014;27(3):339-346. DOI: 10.3122/jabfm.2014.03.130242.

4. Machida M, Nakamura I, Saito R. Changes in implementation of personal protective measures by ordinary Japanese citizens: a longitudinal study from the early phase to the community transmission phase of the COVID-19 outbreak. Int J Infect Dis 2020;96:371-375. DOI: 10.1016/j.ijid.2020.05.039.

5. Coronavirus disease (COVID-19) advice for the public by WHO; 2020. available from: https://www.who.int/emergencies/diseases/novelcoronavirus-2019/advice-for-public. Accessed June 4, 2020.

6. Clinical Management Protocol: COVID 19 by Government of India. Ministry of Health and Family Welfare; 2020. Available from: https:// www.mohfw.gov.in/pdf/ClinicalManagementProtocolforCOVID19. pdf. Accessed June 13, 2020
7. COVID-19 Guidelines For Dental Colleges, Dental Students And Dental Professionals By Dental Council Of India; 2020. Available from: http:// dciindia.gov.in/Admin/NewsArchives/DCI\%20Guidelines\%20on\%20 COVID-19.pdf. Accessed June 16, 2020.

8. Peng X, Xu X, Li Y, et al. Transmission routes of 2019-nCoV and controls in dental practice. Int J Oral Sci 2020;12(1):9. DOI: 10.1038/s41368-0200075-9.

9. Chan TK. Universal masking for COVID-19: evidence, ethics and recommendations. BMJ Glob Health 2020;5(5):e002819. DOI: 10.1136/ bmjgh-2020-002819.

10. Xiao J, Shiu EYC, Gao H, et al. Nonpharmaceutical measures for pandemic influenza in nonhealthcare settings-personal protective and environmental measures. Emerg Infect Dis 2020;26(5):967-975. DOI: 10.3201/eid2605.190994.

11. Fong MW, Gao H, Wong JY, et al. Nonpharmaceutical measures for pandemic influenza in nonhealthcare settings-social distancing measures. Emerg Infect Dis 2020;26(5):976-984. DOI: 10.3201/ eid2605.190995.

12. Johnson DF, Druce JD, Birch C, et al. A quantitative assessment of the efficacy of surgical and $\mathrm{N} 95$ masks to filter influenza virus in patients with acute influenza infection. Clin Infect Dis 2009;49(2):275-277. DOI: $10.1086 / 600041$.

13. Grayson ML, Melvani S, Druce J, et al. Efficacy of soap and water and alcohol-based hand-rub preparations against live H1N1 influenza virus on the hands of human volunteers. Clin Infect Dis 2009;48(3):285-291. DOI: 10.1086/595845.

14. Leung NHL, Chu DKW, Shiu EYC, et al. Respiratory virus shedding in exhaled breath and efficacy of face masks. Nat Med 2020;26(5):676680. DOI: 10.1038/s41591-020-0843-2.

15. Healthy Habits to Help Prevent Flu; 2020. Available from: https:// www.cdc.gov/flu/prevent/actions-prevent-flu.htm. Accessed June 16, 2020. 